\title{
Improvement of multidisciplinary integration in design of complex systems by implementing knowledge-based engineering
}

\author{
Chen Zheng ${ }^{1}$, Matthieu Bricogne ${ }^{1}$, Julien Le Duigou ${ }^{1}$, Peter Hehenberger ${ }^{2}$, Sandor \\ Vajna $^{3}$, Benoît Eynard ${ }^{1}$ \\ ${ }^{1}$ Sorbonne Universités, Université de Technologie de Compiègne \\ Department of Mechanical Systems Engineering, UMR CNRS 7337 Roberval \\ CS 60319, 60203 Compiègne Cedex, France \\ \{chen.zheng; matthieu.bricogne; julien.le-duigou; benoit.eynard\}@utc.fr \\ ${ }^{2}$ Institute of Mechatronic Design and Production \\ Johannes Kepler University Linz, Linz, Austria \\ peter.hehenberger@jku.at \\ ${ }^{3}$ Otto-von-Guericke University Magdeburg \\ Faculty of Mechanical Engineering, 39106, Magdeburg, Germany \\ sandor.vajna@ovgu.de
}

\begin{abstract}
Interface in one complex system represents the logical or physical relationship integrating the components of the complex system or the components with their environment. It plays a quite significant role to guarantee the components designed by the designers of different disciplines integrate correctly in order to achieve the multidisciplinary integration. This paper presents an interface knowledge base to capitalise the design data and rules related to the interfaces. A knowledge-based engineering (KBE) design methodology in which the interface knowledge base is implemented is then proposed to help the designers to improve the multidisciplinary integration during the detailed design phase of complex systems. This KBE design methodology is finally demonstrated by a case study based on a partial discharge (PD) detection system.
\end{abstract}

Keywords: Design methodology, Knowledge-based engineering, Complex systems, Interface model, Multidisciplinary integration

\section{Introduction}

Current development trends indicate that systems become increasingly complex due to the constantly changing requirements and the introduction of new technologies. Pure mechanical systems that generate certain motions or transfer forces or torques, have been used for a long time. Since the beginning of 20th century, control systems have been introduced to manipulate the variables of mechanical systems. This kind of evolution allows fulfilling much more functions compared with a pure mechanical 
system. In 1969, the Yaskawa Electric Corporation coined the term "Mechatronics" as a combination of mechanical systems and electronic systems [1]. After the 1970s, the meaning of Mechatronics has been broadened to include software and computation. From then on, more disciplines, such as optical, hydraulic, pneumatic, telecommunication disciplines, etc., have been gradually integrated into products and systems. During the 1990s, driven by the development of information and communication technologies, systems are influenced by some new development trends shifting towards information processing associated with personal computers processing [2]. In this context, various types of complex systems, such as embedded systems, Cyber-Physical Systems (CPS), Internet of Things (IoT), Systems of Systems (SoS), etc., have been proposed and studied.

The above introduction reveals that the design of complex systems encompasses a wide range of disciplines. Therefore increasing attention has been paid to the multidisciplinary integration. The multidisciplinary integration not only requires the synergic integration of involved disciplines, but also focuses on the designers' activities of the whole design process [3]. During the detailed design phase of complex systems, components are usually designed in parallel by designers of different disciplines.

The interface is at the heart of the multidisciplinary nature for the design of complex systems [4]. The interface in the complex systems refers to the logical or physical relationships integrating the components of one system or the components with their environment, which can be used to describe the interactions of the components designed by different disciplines [5]. This paper presents a knowledgebased engineering (KBE) methodology, in which the interface model is considered as the key part to help the designers to improve the multidisciplinary integration. Two new features are provided by the proposed KBE design methodology. First, it has the capability to capture and organise the design data related to the interface and store the data in a structured base in order to achieve the reuse of knowledge. Second, it provides an automated compatibility test to guarantee the correction of different components. By applying the two features proposed by the KBE design methodology, both the synergistic integration of the components in one complex system and the integration of the several involved disciplines during the design process become more integrated, so that the multidisciplinary integration of complex systems can be improved.

The paper is organised as follows. Section 2 presents a review of related work. Section 3 firstly introduces the interface model which is considered as the key part of the proposed KBE design methodology. Then, an overview of the proposed KBE design methodology is presented. A case study based on a partial discharge (PD) detection system will be introduced in Section 4 to demonstrate the proposed KBE design methodology. Finally, the authors draw the conclusions in Section 5.

\section{Related work}

The design of complex systems in which several disciplines are involved requires the extension of knowledge used by to include more disciplines. In order to achieve 
the multidisciplinary integration, some multidisciplinary design methodologies have been presented. Many researchers focus on the methodologies based on CAD to help the designers for the multidisciplinary design. For example, Lefèvre et al [6] extract the geometric information from a CAD representation of the control component of a motor fan to simulate the dissipation by Ansys Fluent. Biahmou et al [7] propose a methodology to translate a CAD model into behaviour simulation models created in MATLAB/Simulink. The methodologies based on CAD only focus on the product dimensions, geometric and surface contour and product materials, but neglect the design data and rules that can be reused during the detailed design phase [8].

$\mathrm{KBE}$ has some origins in $\mathrm{CAD}$, and moreover, it can be used to capture and apply discipline-specific knowledge and expertise in order to facilitate solving problems [9]. Therefore KBE has been widely adopted for the design of discipline-specific systems, especially in mechanical engineering discipline. Sapuan and Abdalla [9] develop knowledge-based system for the material selection for the design of mechanical systems, Chapman and Pinfold [10] describe an application of a KBE approach for the rapid design and analysis of mechanical $\mathrm{s}$ of automobiles and Yang et al [11] propose a KBE methodology for the design of ship decks. As to other disciplines, $\mathrm{KBE}$ can also be used to assist the design process. Aurum et al [12] point out the importance of knowledge during the software engineering process and propose a structured model to manage the software engineering knowledge. In the electronic engineering discipline, Wang et al [13] discuss the possibility to apply KBE to the rapid design of process chambers of integrated circuits.

This literature review shows that KBE can be used as an effective support for the discipline-specific design. Nowadays, by considering the specialities of the multidisciplinary design, the KBE has been gradually implemented in the design of complex systems. Chen et al [14] propose a knowledge-based framework for the conceptual design phase of multidisciplinary systems. La Rocca and van Tooren [15] develop conceptual aircraft design applications based on KBE. Tian and Voskuijl [16] integrate the knowledge related to Electronic systems into the application developed by La Rocca and van Tooren to support the conceptual design of mechatronic systems. The literature review shows that most of the studies on those design methodologies based on KBE focus on the conceptual design phase. How to achieve the collaboration of designers from different disciplines during the detailed design phase has not been discussed.

Considering the limitations of related works on the multidisciplinary integration for the design of complex system, the authors propose a KBE design methodology in which the interface model is implemented to help the designers to improve the multidisciplinary integration during the detailed design phase for complex systems. Next section will present the details of the proposed design methodology.

\section{$3 \quad$ KBE design methodology}

The KBE design methodology presented in the paper implements the interface model as the key part to help the designers to improve the multidisciplinary integration during the detailed design phase of complex systems. The knowledge base 
is used to collect the design data, rules, experiences and other knowledge. In the paper, the authors propose a knowledge base which stores the design data and rules related to the interface by instantiating the interface model. This interface model will be firstly introduced in this section.

\subsection{Interface model}

The authors propose the interface model to deal with the problem of multidisciplinary integration [17]. This interface model not only provides a structured base to store the design data related to the interface in order to achieve the knowledge reuse, but also offers compatibility rules to the designers in order to guarantee that different components integrate correctly (Fig. 1).

First, the proposed interface model provides a structured base to store the design data related to the interface in order to achieve the knowledge reuse. It contains classes to define the attributes of one interface and its ports. The term "port" is considered as the primary location through which one element of a system interacts with other elements. The interface attributes are defined by taking into consideration of three different features: type, configuration and desired/undesired. Type attribute focuses on which types of transfer (geometric, energy, control or data) occur through one interface. Configuration attribute describes which elements are linked by the interface. Desired/undesired attribute expresses whether the interface creates positive effects (e.g. data or energy transmission) or unintended side-effects (e.g. heat, magnetic fields, vibration and other side effects. In summary, the attributes contained in the interface model provide a common representation for the interfaces defined by design teams of different disciplines.

Second, the interface model offers the compatibility rules to the designers to guarantee the different components integrate correctly. The method compatibility () is contained by the class Interface to check the compatibility of the interface. One example is cited here to illustrate the compatibility test method. Two components (Component 1 and Component 2) are connected by an interface (Interface) through the ports (CP1 and CP2). Two compatibility rules are presented as follows.

$$
\begin{gathered}
\text { CP1. Parameters } 1 . \text { value }=C P 2 \text {. Parameters } 2 . \text { value } \\
\text { CP1. Parameters } 1 . \text { unit }=C P 2 . \text { Parameters } 2 \text {. unit }
\end{gathered}
$$

$$
\begin{aligned}
& \text { CP1. Parameters1. value }<\text { CP2. Parameters } 2 \text {. maxValue } \\
& \text { CP1.Parameters1. value }>\text { CP2. Parameters } 2 \text {. minValue } \\
& \text { CP1. Parameters } 1 \text {. unit }=\text { CP2. Parameters } 2 \text {. unit }
\end{aligned}
$$

CP1.Parameter1 represents the parameter stored in the class Parameter of the port CP1, and CP2.Parameter2 is the parameter of port CP2. In the compatibility Rule 1, in order to ensure the two components integrate with each other correctly, both the value and the unit of the parameters of CP1 and CP2 should be equal. However, sometimes the design parameter of one port is not specified by an exact value accurately. The compatibility Rule $\mathbf{2}$ is used to illustrate that case. If the port CP2 specifies the parameter by using an interval (minValue, maxValue), the parameter of CP1 should satisfy that CP1.Parameter1.valueE (CP2. Parameters2.minValue, CP2.Parameters2.maxValue). 
The interface knowledge base being dealt in this paper is based on the previous interface model. Next sub-section will present the interface knowledge base and the KBE design methodology based on the interface knowledge base.

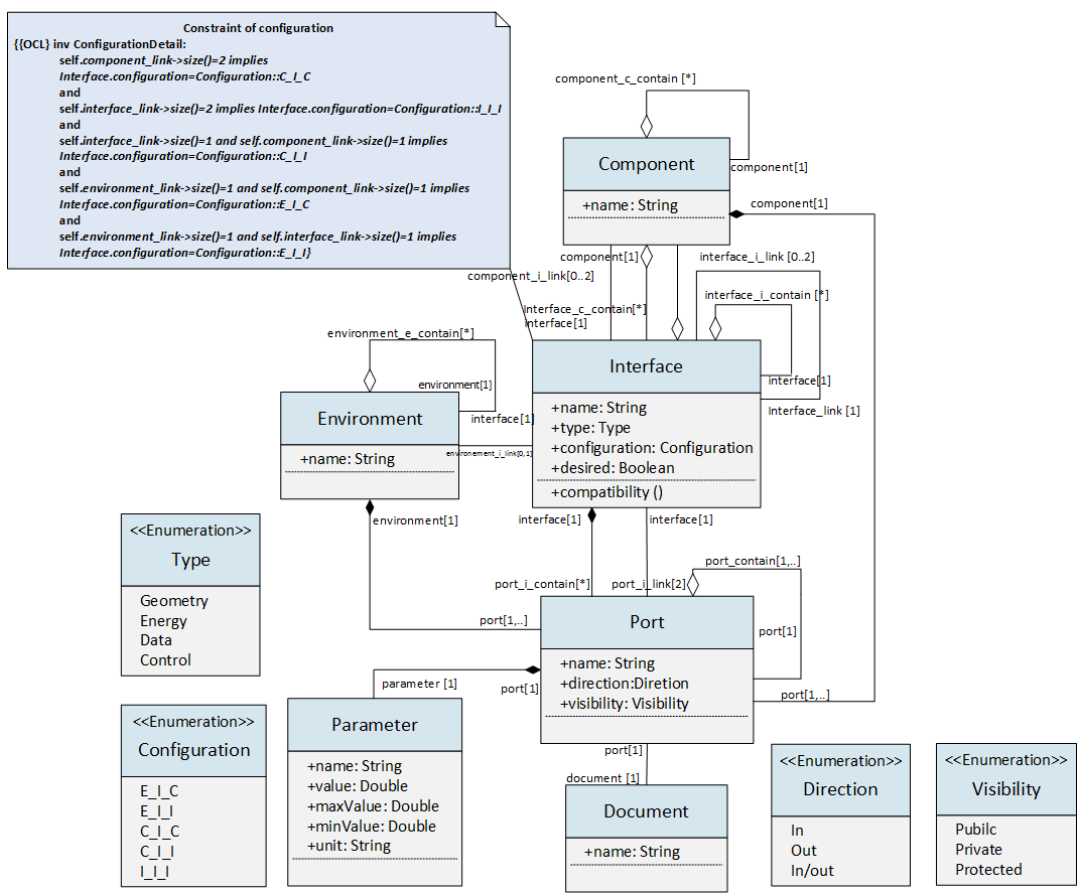

Fig. 1. UML class diagram of interface

\subsection{Interface knowledge base in detailed design phase}

In this paper, a knowledge-based engineering approach is applied to the detailed design phase of complex systems. The designers should capitalise the design input parameters including the interface data and compatibility rules from the heterogeneous and multidisciplinary sources and store them into the interface knowledge base. The flow chart based on the interface knowledge base is shown in Fig. 2, which mainly illustrates how the knowledge-based engineering approach can help the designers to improve the multidisciplinary integration during the detailed design phase.

Before entering the detailed design phase, the system designers have defined the architecture of the complex system [18]. In other words, the system designers should decompose the architecture recursively until the standard components or the components that have to be designed by the designers of discipline-specific teams. At the beginning of the detailed design phase, the designers of different disciplines carry out their design process for the discipline-specific components. The designers capture the design data and capitalise the design rules related to the interfaces generated or proposed during the design process of discipline-specific components. Then the 
interface model is instantiated by the design data and the rules and stored in the interface knowledge base. Once the model of an interface has been instantiated and stored in the interface knowledge base, the interface compatibility should be checked by using the compatibility rules in order to guarantee the different components integrate correctly. If the components prove to be incompatible with each other, the iterative process should be carried on. In the iterative process, the designers take the design data stored in the interface knowledge base to redesign the components in order to solve the incompatibility problems.

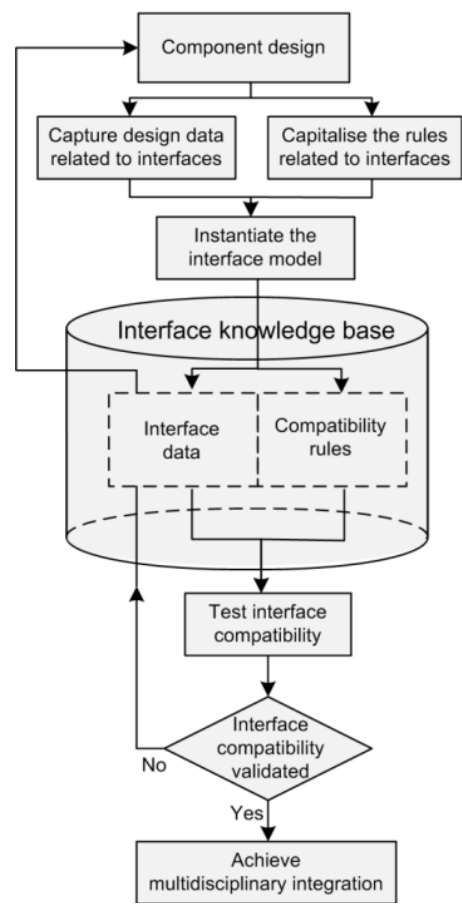

Fig.2. Flow chart of detailed design phase based on interface knowledge base

In this section, the interface knowledge base and the design methodology based on it have been presented. Knowledge bases are generally presented as a way to store design knowledge such as design data and design rules. The interface knowledge promotes the multidisciplinary integration by ensuring that the interfaces compatibility rules are respected. A case study is proposed by means of a partial discharge (PD) detection system to demonstrate the KBE methodology.

\section{Case study}

The case study chosen to demonstrate the KBE methodology in this section is a partial discharge (PD) detection system. This PD detection system is designed to detect the partial discharge in gas insulated switchgear (GIS). GIS is a new type of 
high voltage switchgear that has been widely used in electric power systems. Although the reliability of GIS is high, any insulation defect that occurs without warning may result in damage to neighbouring equipment, customer dissatisfaction, disruption to economic activity, and the imposition of regulatory fines [19]. The PD detection system has been widely applied in GIS to detect the partial discharge in the GIS in order to avoid the risk of insulation accident. The PD detection system is considered as a complex system integrating synergistically the electrical/electronic system, mechanical parts, information processing and telecommunication technology, so the design of the system requires a multidisciplinary integration. Moreover, PD detection system has a number of variants according to the different internal structures or the voltage classes of the GIS. Similar design of the variants can directly reuse the knowledge base including the design data and rules, which can significantly shorten the design cycle to achieve the goal of rapid design and improve the quality, efficiency of design and production. Fig. 3 shows the principle of the PD detection system. The PD signal can be captured by an ultra-high frequency sensor and transferred by the coaxial transmission line to the signal pre-processing sub-system where the analogue PD signal will be filtered, amplified and eventually converted to the digital data. Such digital data will be acquired and further processed. The processing results will be used for diagnostic purposes and control the operation state of the GIS.

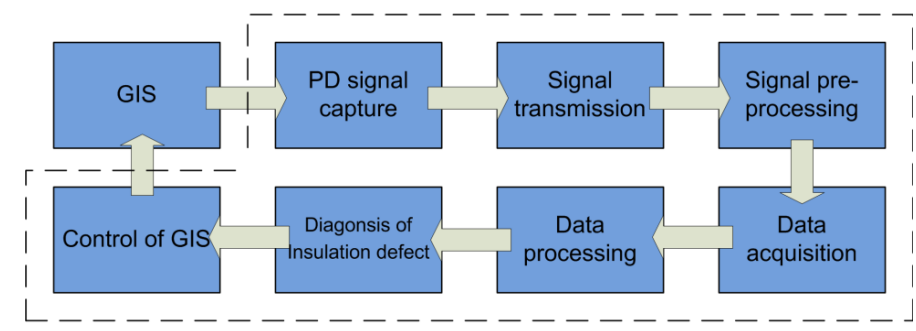

Fig.3. Principle of PD detection system

The principle of the PD detection system indicates that different disciplines are involved in the design process. For example, the ultra-high frequency sensor to capture the PD signal is designed by the telecommunication team, whereas the coaxial wave line and the signal pre-processing sub-system are chosen and designed by the electronic team. Therefore the integration of the ultra-high frequency sensor and the coaxial transmission line will be chosen in order to demonstrate the proposed KBE based design methodology more clearly.

The ultra-high frequency sensor plays a crucial role to the accuracy and sensitivity of detection in the PD detection system [19]. It is designed by the designers of telecommunication team due to their rich experiences in antenna design. The designers of telecommunication team adopt the planar equiangular spiral antenna (PESA) as the ultra-high frequency sensor to capture the PD signal due to its ultra wideband feature. By considering the internal structure of the GIS and the electromagnetic wave's bandwidth created by the PD signal, the designers design the PESA with bandwidth from $700 \mathrm{MHz}$ to more than $3 \mathrm{GHz}$, and its outside radius is 
$109 \mathrm{~mm}$ and initial radius is $2 \mathrm{~mm}$. The software ANSYS ${ }^{1}$ is used for modelling and simulation of the PESA, and the simulation result shows that the impedance of the antenna is about $135 \Omega$. The designers of the electronic team choose the standard coaxial transmission line to transfer the PD signal captured by the ultra-high frequency sensor, whose impedance is $50 \Omega$.

The demonstrator based on the KBE methodology has been developed by making use of the 3DEXPERIENCE platform ${ }^{2}$. The two components, i.e., the ultra-high frequency sensor and the coaxial transmission line (two blue boxes) and the interface between them (purple box) are presented by the demonstrator on the top of Fig.4. The UML class diagram below the interface I1 in Fig. 4 shows the instance of the interface by considering the design data related to the interface. The attributes of the interface I1 and the ports' parameters linked to I1 are transferred into a structured representation by the demonstrator (shown on the left side and at the bottom of Fig.4) and stored in the Interface knowledge base (shown in the centre of Fig.4). The rule to check the compatibility of the interface I1 is processed by the Knowledgeware workbench of the 3DEXPERIENCE platform. The check result of interface compatibility (shown on the right side of Fig.4) indicates that the interface between the ultra-high frequency sensor and the coaxial transmission line is incompatible, because the impedance of the ultra-high frequency sensor is different from that of the coaxial transmission line. The difference of the impedances between the ultra-high frequency sensor and the coaxial transmission line implies that the two components do not match with each other, which affects the transmission of the PD signal negatively [20].

The flow chart in Fig. 2 shows that if the components prove to be incompatible with each other, the iterative process should be carried on. The designers should consider the design data related to the incompatible interface stored in the interface knowledge base. A micro-strip impedance transformer is designed by the designers of the telecommunication team which can change the impedance from $135 \Omega$ to $50 \Omega$ will be added between the ultra-high frequency sensor and the coaxial transmission line. After applying the incompatibility problem solution during the iterative process, a micro-strip impedance transformer is added between the ultra-high frequency sensor and the coaxial transmission line.

\section{Conclusion}

This paper presents a KBE methodology to help the designers to improve the multidisciplinary integration during the design process of complex systems. The interface knowledge base that is constructed and based on the interface model is implemented in the KBE methodology. It not only has the capability to capture the design data related to the interface and to store them in a structured base in order to achieve the knowledge reuse, but it provides an automated compatibility test to guarantee the different components integrate correctly as well. A PD measurement

\footnotetext{
1 http://www.ansys.com/

${ }^{2} \mathrm{http}: / /$ www.3ds.com/about-3ds/3dexperience-platform/
} 
system has been chosen as case study to demonstrate how the KBE methodology can support efficiently the detailed design phase of complex systems by making use of a demonstrator developed based on the 3DEXPERIENCE platform. By applying the two features proposed by the KBE based design methodology, both the synergistic integration of the components in one complex system and the integration of the several involved disciplines during the design process will become more integrated, so that the multidisciplinary integration of complex systems can be improved.

The literature review in Section 2 has shown that some design applications based on the KBE have been developed for the conceptual design phase of multidisciplinary systems; while the KBE methodology proposed in the paper focuses on the detailed design phase. However, little attention has been paid to the link between the conceptual design phase and the detailed design phase, i.e., the system architecture definition. How to use the KBE approach to support the architecture definition should be further studied in the future.

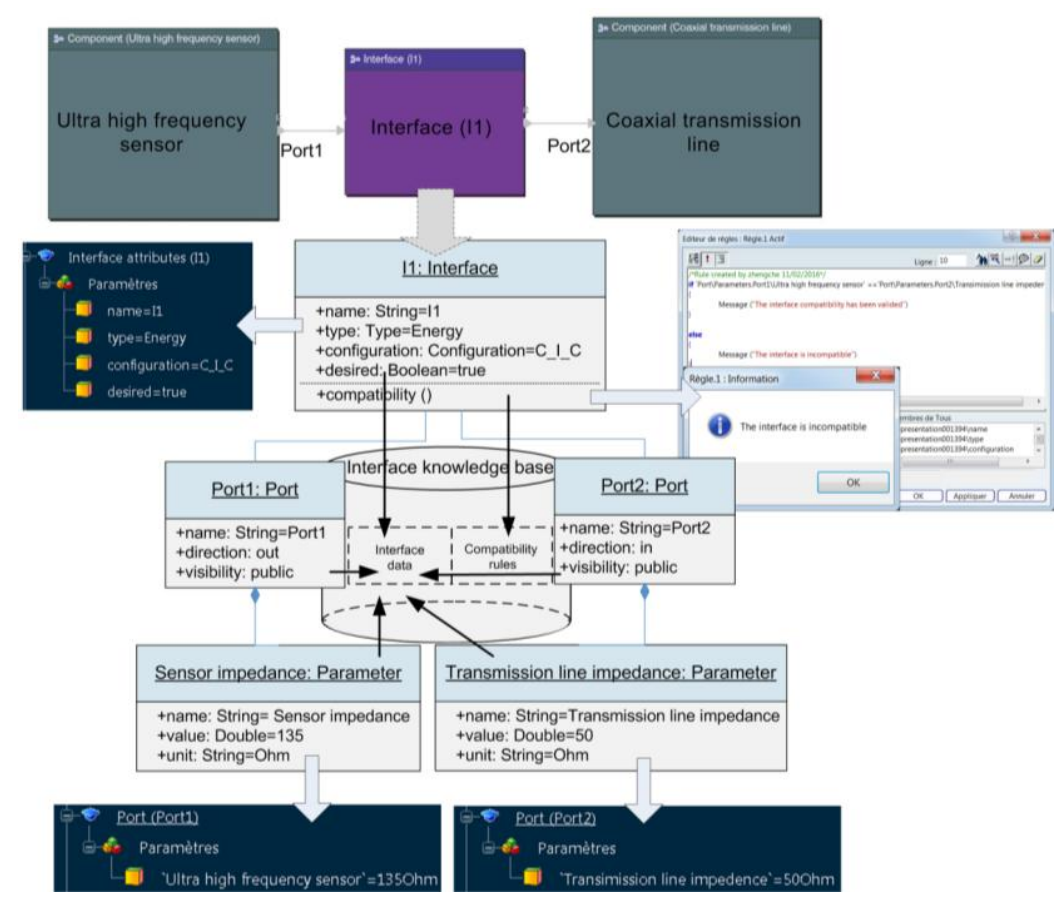

Fig.4. Instantiation of the interface model and interface knowledge base

Acknowledgement. This work has been partially supported by the Doctoral Program of Chinese Scholarship Council and the Austrian Center of Competence in Mechatronics (ACCM) / Linz Center of Mechatronics (LCM) in the framework of the Austrian COMET program. It also takes place in the scientific strategy of Labex MS2T supported by the ANR - French National Agency for Research. 


\section{References}

1. Carryer, J.E., Ohline, R.M., Kenny, T.W.: Introduction to Mechatronic Design. Prentice Hall, Boston (2011)

2. Marwedel P.: Embedded System Design: Embedded Systems Foundations of Cyber-Physical Systems. Springer, Dordrecht (2011)

3. Zheng, C., Bricogne, M., Le Duigou, J., Eynard, B.: Survey on Mechatronic Engineering: a Focus on Design Methods and Product Models. Adv Eng Informatics. 28:241--57 (2014)

4. Fosse, E., Delp, C.L.: in:, Systems Engineering Interfaces : a Model Based Approach. 2013 IEEE Aerospace Conference, Big Sky (2013)

5. Liang, V.C., Paredis, C.J.J.: A Port Ontology for Conceptual Design of Systems. J. Comput. Inf. Sci. Eng. 4, 206--217 (2004)

6. Lefèvre, J., Charles, S., Bosch-Mauchand, M., Eynard, B., Padiolleau, E.: Multidisciplinary Modelling and Simulation for Mechatronic Design. J. Des. Res. 12, 127--144 (2014)

7. Biahmou, A., Frohlich, A., Stjepandic, J.: Improving Interoperability in Mechatronic Product Development. in: Proceedings of the International Conference on Product Lifecycle Management, Bremen (2010)

8. Kulon, J., Broomhead, P., Mynors, D.J.: Applying Knowledge-based Engineering to Traditional Manufacturing Design. Int. J. Adv. Manuf. Technol. 30, 945--951 (2006)

9. Sapuan, S.M.: A Knowledge-based System for Materials Selection in Mechanical Engineering Design. Mater. Des. 22, 687--695 (2001)

10. Chapman, C.B., Pinfold, M.: The Application of a Knowledge Based Engineering Approach to the Rapid Design and Analysis of an Automotive Structure. Adv. Eng. Softw. 32, 903--912 (2001)

11. Yang, H.Z., Chen, J.F., Ma, N., Wang, D.Y.: Implementation of Knowledge-based Engineering Methodology in Ship Structural Design. Comput. Des. 44, 196--202 (2012)

12. Aurum, A., Jeffery, R., Wohlin, C., Handzic, M.: Managing Software Engineering Knowledge, Springer Berlin Heidelberg, Heidelberg (2013)

13. Wang, K.S., Lin, J., Cheng, J., Ji, L.H.: Investigation on the Development of Knowledgebased Engineering and Its Application in Rapid Design of Process Chamber of IC Equipment. Appl. Mech. Mater. 373, 2147-- 2155 (2013)

14. Chen, Y., Liu, Z.L., Xie, Y.B.: A Knowledge-based Framework for Creative Conceptual Design of Multi-disciplinary Systems. Comput. Des. 44, 146--153 (2012)

15. La Rocca, G., van Tooren, M.J.: Knowledge-based Engineering to Support Aircraft Multidisciplinary Design and Optimization. J. Aerosp. Eng. 224, 1041--1055 (2010)

16. Tian, F., Voskuijl, M.: Mechatronic Design and Optimization Using Knowledge Based Engineering Applied to an Inherently Unstable and Unmanned Aerial Vehicle. IEEE/ASME Trans. Mechatronics. DOI:10.1109/TMECH.2015.2441832 (2015)

17. Zheng, C., Le Duigou, J., Bricogne, M., Eynard, B.: Multidisciplinary Interface Model for Design of Mechatronic Systems. Comput. Ind. 76, 24--37 (2016)

18. Sage, A.P., Rouse, W.B.: Handbook of Systems Engineering and Management, John Wiley \& Sons, Hoboken (2009)

19. Judd, M.D., Yang, L., Hunter, I.B.B.: Hunter, Partial Discharge Monitoring of Power Transformers using UHF sensors. IEEE Electr. Insul. Mag. 21, 5--14 (2005)

20. Zheng, C., Li, T.: Development of an Exponential Tapered Impedance Transformer for UHF-PD Sensor. In: 1st International Conference on Electric Power Equipment, Xi'an (2011) 